\title{
Effects of Postponing Treatment in the Second Year of Cladribine Administration: Clinical Trial Simulation Analysis of Absolute Lymphocyte Counts and Relapse Rate in Patients with Relapsing-Remitting Multiple Sclerosis
}

\author{
Nadia Terranova $^{1} \cdot$ Christine Hicking $^{2} \cdot$ Fernando Dangond $^{3} \cdot$ Alain Munafo $^{1}$
}

Published online: 11 July 2018

(c) The Author(s) 2018

\begin{abstract}
Introduction Cladribine Tablets (MAVENCLAD ${ }^{\circledR}$ ) selectively reduce absolute lymphocyte counts (ALCs) in patients with multiple sclerosis. The recommended cumulative dose of Cladribine Tablets is $3.5 \mathrm{mg} / \mathrm{kg}$ over $4-5$ days in months 1 and 2 of treatment years 1 and 2, followed by prolonged efficacy with no additional treatment. After the cladribine-induced reduction, ALCs recover to normal within each treatment year in most patients. Those patients with slow ALC recovery can develop Grade 3-4 lymphopenia, especially those patients with Grade $\geq 2$ lymphopenia at the start of year 2 . Guidelines allowing treatment postponements during year 2 have been proposed for patients with a low ALC, subsequent to CLARITY, the pivotal clinical trial.

Methods A virtual population was generated using characteristics from CLARITY patients. A clinical trial simulation was performed to determine the impact of alternative treatment scenarios on ALC and relapse rate, by postponing treatment in year 2 to allow for longer ALC recovery time in patients who required it. Should a patient not recover to normal ALC (Grade 0 ) or Grade 1 lymphopenia within the period defined in the treatment algorithm, treatment in year 2 was suspended. Results Results were similar across considered scenarios, which implemented different postponement durations. Specifically, $\sim 92 \%$ of virtual subjects did not require treatment postponement and $<1 \%$ discontinued due to Grade 2-4 lymphopenia at the end of the maximally permitted postponement. Less severe lymphopenia was observed during year 2 when a treatment algorithm was applied. The effect on relapse rate over 2 years was negligible.

Conclusions Results support treatment guidelines to decrease the risk of severe lymphopenia following treatment with Cladribine Tablets, while preserving efficacy.
\end{abstract}

Trial Registration CLARITY; ClinicalTrials.gov: NCT00213135.

Both the Merck Institute for Pharmacometrics (Merck Serono S.A.), Switzerland and EMD Serono Inc., USA, are affiliates of Merck KGaA, Darmstadt, Germany.

Electronic supplementary material The online version of this article (https://doi.org/10.1007/s40262-018-0693-y) contains supplementary material, which is available to authorized users.

Nadia Terranova

nadia.terranova@merckgroup.com

1 Quantitative Pharmacology, Merck Institute for Pharmacometrics, Merck Serono S.A., EPFL Innovation Park - Building I, CH-1015 Lausanne, Switzerland

2 Global BioStatistics, Merck KGaA, Darmstadt, Germany

3 Global Clinical Development - Neurology, EMD Serono Inc., Billerica, MA, USA 


\section{Key Points}

This clinical trial simulation was performed using a virtual population based on characteristics from the CLARITY study to determine the impact of alternative treatment scenarios on absolute lymphocyte count (ALC) and relapse rate.

Our results indicate that $92 \%$ of patients will not require their treatment to be postponed, but by allowing a 6-month delay, 99\% of patients would be eligible for their second-year course of treatment.

Implementation of ALC-based treatment guidelines would reduce the occurrence of Grade 3-4 lymphopenia at any time, without having a negative impact on the risk of relapse over 2 years.

\section{Introduction}

Multiple sclerosis is a chronic disorder of the central nervous system, where lymphocyte-driven inflammation causes demyelination and axonal damage, ultimately resulting in progressive disability [1]. Cladribine Tablets (MAVENCLAD ${ }^{\circledR}$; Merck Serono Europe Ltd) were recently approved in Europe for the treatment of adults with highly active relapsing multiple sclerosis [2]. The mechanism of action of cladribine, a nucleoside analogue of deoxyadenosine, is the selective depletion of $\mathrm{B}$ and $\mathrm{T}$ lymphocytes.

Due to the mechanism of action, lymphopenia is an expected event after treatment with Cladribine Tablets. However, severe lymphopenia is associated with an increased risk of infections [3] and should be avoided. In the pivotal phase III CLARITY (CLAdRIbine Tablets treating multiple sclerosis orallY) study, lymphopenia severity was graded using the Common Terminology Criteria for Adverse Events (CTCAE) version 3.0 [4]. Grade 3 and 4 lymphopenia at any time in the study was observed in $24.9 \%$ (107/430) and $0.7 \%$ (3/430) of patients, respectively, during treatment with a $3.5 \mathrm{mg} / \mathrm{kg}$ cumulative dose of Cladribine Tablets over 2 years [5].

The recommended cumulative dose of Cladribine Tablets is $3.5 \mathrm{mg} / \mathrm{kg}$ body weight (BW) over 2 years, administered as one treatment course of $1.75 \mathrm{mg} / \mathrm{kg}$ per year. Each treatment course consists of two treatment weeks, one at the beginning of the first month and one at the beginning of the second month of the respective treatment year. Each treatment week consists of 4 or 5 days on which a patient receives 10 or $20 \mathrm{mg}$ as a single daily dose, depending on BW [2]. Cladribine Tablets have a durable effect, with clinical data showing that the proportion of patients free from sustained disability progression was maintained for more than 2 years after the last dose of active treatment [6].

The overall objective of the clinical trial simulation work presented herein was to investigate the impact of alternative treatment rules driven by sustained lymphopenia at the beginning of the second year on the occurrence of qualifying relapses in patients with relapsing-remitting multiple sclerosis (RRMS) versus nominal treatment rules for Cladribine Tablets. The nominal dosing strategy in this analysis was no postponement of treatment in the second year regardless of the patient's absolute lymphocyte count (ALC), with the alternative rules imposing a range of postponement durations to allow for ALC recovery before the second year of treatment. Two previously developed population pharmacodynamics (popPD) models, namely a repeated time-to-event (RTTE) model of relapse rate (RR) and an ALC dynamics model, were used to obtain projections for RR and ALC following treatment postponements or suspension [7].

\section{Methods}

\subsection{Patient Population}

A virtual population was generated by considering participants of the CLARITY trial [5] who received a $3.5 \mathrm{mg} /$ $\mathrm{kg}$ cumulative dose of Cladribine Tablets, as described in Sect. 2.2. This 'CLARITY scenario', which consisted of 2 annual courses that were each comprised of 2 treatment weeks -1 at the start of the first month and 1 at the start of second month of each year-was considered as the reference scenario. Full details of the dosing schedule used in the CLARITY study have been reported previously [5, 8]. A qualifying relapse was defined as an increase of 2 points in at least 1 functional system of the Expanded Disability Status Scale or an increase of 1 point in at least 2 functional systems (excluding changes in bowel or bladder function or cognition) in the absence of fever, lasting for $\geq 24 \mathrm{~h}$ and preceded by $\geq 30$ days of clinical stability or improvement [5].

To generate a virtual population that would comply with the risk-minimisation measure of not initiating Cladribine Tablets in year 1 (Y1) in patients with lymphopenia, patients with a baseline ALC below $1 \times 10^{9}$ cells/L (i.e. Grade $1-4$ lymphopenia) were excluded from the analysis of the base scenario and treatment scenarios. This exclusion criterion was not applied to the original CLARITY scenario, primarily used to validate the workflow, in order to accurately represent observations at baseline in the CLARITY trial (where this risk-minimisation measure had not been enforced). 


\subsection{Covariate Distribution Model}

Patient characteristics that were previously identified as significant explanatory covariates, and included in the pharmacodynamic models as a covariate effect, were sampled from distributions observed in the CLARITY study $(N=1319$, including patients from all treatment arms) and assigned to each virtual patient. Covariance among considered characteristics was taken into account.

As sex was found to be a significant explanatory covariate for the parameter describing cladribine potency in the ALC model [7], distributions of such patient characteristics were assessed by sex to ensure that sampled covariates would be physiologically realistic and consistent with observations. Then, for each considered subgroup, the number of relapses in the 12 months preceding study entry (referred to as EXNB) associated with each virtual patient was sampled by considering the observed proportions. Similarly, creatinine clearance $\left(\mathrm{CL}_{\mathrm{CR}}\right)$ and $\mathrm{BW}$ were sampled from a multivariate log-normal distribution characterised by mean values and dispersion measures from distributions observed within each subgroup.

\subsection{Simulation Workflow}

The previously developed popPD models for ALC and RR were used to simulate the ALC and RR dynamics for each patient in the virtual population [7]. A four-step simulation workflow was used:

1. Exploratory and graphical analyses were performed to identify possible correlations between ALC and RR parameters, and to assess whether uncertainty had to be taken into account in case of a low precision of parameter estimates.

2. Patient covariates were generated for the virtual population by considering the distributions observed in CLARITY.

3. As a validation step, the clinical trial simulation was run to reproduce the CLARITY scenario by considering an initial population of 5000 patients, which was found to be a suitable number to reproduce the CLARITY population in terms of the proportions of patients within each lymphopenia grade as well as proportions of virtual patients not experiencing a certain number of relapses.

4. The clinical trial simulation was then performed to simulate alternative treatment rules.

\subsection{Input-Output Model}

The simulation workflow included the model describing ALC dynamics and the RTTE model of RR [7]. An overview of these models is also provided in Electronic Supplementary Material Online Resources 1 and 2. An exploratory analysis was performed to first assess whether any correlation between models had to be taken into account. According to the decision tree shown in Online Resource 3, Figure 1, the precision of fixed-effect parameter estimates was also assessed for the 2 models. Based on results from this step, the 2 models were then used to perform the simulations. Specifically, for each tested scenario, ALC dynamics of virtual patients were simulated by applying dosing algorithms. Corresponding projections of RR were then obtained for each scenario to assess the impact of the individual dosing algorithms on the occurrence of qualifying relapses.

\subsection{Trial Execution Model}

No deviations (e.g. patient non-compliance, patient dropout) from the CLARITY study protocol were considered or reproduced in the simulated scenarios.

\subsection{Treatment Algorithms}

ALC and RR dynamics were simulated for each virtual patient according to predefined treatment rules. In the base scenario (nominal dosing regimen), all virtual patients received 2 treatment courses of Cladribine Tablets $1.75 \mathrm{mg} /$ $\mathrm{kg}$ per year for a cumulative dose of $3.5 \mathrm{mg} / \mathrm{kg}$. Each treatment course consisted of 2 weekly treatments: 1 at the beginning of the first month and 1 at the beginning of the second month of the respective treatment year. The difference between the CLARITY (reference) scenario described in Sect. 2.1 and the base scenario described here was the definition of year duration, which was 48 and 52 weeks, respectively.

Three dosing algorithms with different definitions of postponement blocks were investigated, with the first weekly treatment of year 2 (Y2) being postponed if the patient had Grade 2-4 lymphopenia at the start of Y2. For each scenario, ALC dynamics were simulated at the patient level by allowing individualised treatment postponements during $\mathrm{Y} 2$ according to the rules described in Table 1. Regardless of whether the first weekly treatment of Y2 had to be postponed due to lymphopenia or not, the subsequent treatment week always followed it by 1 month. Treatment was stopped in a given virtual patient if, after 3 postponements, his/her ALC had not recovered to within a lymphopenia range of Grade $0-1$. Additional scenarios allowing postponements only in patients with Grade 3-4 lymphopenia were also explored (data not shown).

Hence, individual dosing regimens resulting from applying treatment rules were used to obtain the resulting occurrence of qualifying relapse for each virtual patient, and then 
Table 1 Treatment algorithms to postpone treatment at the start of year 2 in patients with Grade 2-4 lymphopenia

\begin{tabular}{lll}
\hline $\begin{array}{l}\text { Treatment } \\
\text { scenario }\end{array}$ & $\begin{array}{l}\text { Postponement block } \\
\text { duration (months) }\end{array}$ & $\begin{array}{l}\text { Maximum post- } \\
\text { ponement duration } \\
\text { (months) }\end{array}$ \\
\hline 1 & 1 & 3 \\
2 & 2 & 6 \\
3 & 3 & 9 \\
\hline
\end{tabular}

Lymphopenia graded by the National Cancer Institute Common Terminology Criteria for Adverse Events version 3.0 [13]; Grade 2: 0.5 to $<0.8 \times 10^{9}$ cells/L; Grade 3: 0.2 to $<0.5 \times 10^{9}$ cells/L; Grade 4: $<0.2 \times 10^{9}$ cells $/ \mathrm{L}$

${ }^{a}$ Maximum delay was three blocks in each scenario

derive the predicted time-varying probability distribution of relapse-free survival in the virtual population.

\subsection{Software}

The statistical package R (version 3.2.1; https://www.r-proje ct.org) was used in the analysis. The model simulations themselves were performed using the mlxR 2.2.0 package [9], with models encoded in Mlxtran [10].

\section{Results}

\subsection{Patient Characteristics}

In total, 1319 observations were used to determine the distribution and covariance among patient characteristics included in the models as covariates. In the CLARITY study, $32 \%$ of patients were male and 68\% were female [5]. Mean (standard deviation [SD]) $\mathrm{CL}_{\mathrm{CR}}$ was $114.65(0.22) \mathrm{mL} / \mathrm{min}$ for male patients and $101.96(0.26) \mathrm{mL} / \mathrm{min}$ for female patients in the observed CLARITY population. Mean (SD) BW was $75.02(0.17) \mathrm{kg}$ for observed male patients and $64.54(0.21)$ $\mathrm{kg}$ for observed female patients. The correlation between observed $\mathrm{CL}_{\mathrm{CR}}$ and $\mathrm{BW}$ was 0.645 and 0.680 in male and female patients, respectively. The probabilities associated with experiencing between 0 and 5 relapses in the preceding 12 months (EXNB) ranged from 0 for no relapses in the previous year to 0.7 for 1 relapse in the previous year. The virtual population was first generated by maintaining the observed proportions of male and female patients. Then, covariates $\left(\mathrm{EXNB}, \mathrm{CL}_{\mathrm{CR}}\right.$ and $\left.\mathrm{BW}\right)$ were sampled for each subgroup for a total population size of 5000 virtual patients. Sampled characteristics closely mimicked those observed for both male and female patients in the CLARITY study (Fig. 1b). The EXNB distributions were also very similar to those observed in the 2 subgroups (Fig. 1a). By applying the exclusion criteria described in Sect. 2.1, a total of 180 subjects with baseline ALC below $1 \times 10^{9}$ cells/L (i.e. Grade 1-4 lymphopenia) were excluded from the virtual population, which was used in the base and alternative treatment scenarios.

\subsection{Model Parameters}

The adoption of the models for ALC and RR was based on the decision tree presented in Electronic Supplementary Material 3, Figure 1, where potential correlations between models and precision of parameter estimates were assessed.

As no dependency (correlation less than 30\%) among the estimated random effects of the 2 models was observed, no further complexities had to be considered at this stage and the models could be used separately. Subsequently, based on the decision tree, fixed-effect parameters and inter-individual variability parameters of the model for ALC were fixed to the final estimates of the previously developed model, given that less than 2 fixed-effect parameter estimates showed poor precision (percentage relative standard error above 25\%). In contrast, parameter uncertainty was taken into account in the RTTE model for RR by resampling the parameters from a normal distribution with mean set to point parameter and dispersion to the variance-covariance matrix precision of those parameter estimates. The obtained parameter values were then used to perform model simulations for RR. Individual parameters obtained from both model simulations were preserved across scenarios. No intra-patient variability or assay variability were considered.

\subsection{Evaluation of Simulation Framework: CLARITY Scenario}

The 5000-patient virtual population (more than 10 times larger than the original sample) was found to be a suitable size to reproduce the target population of CLARITY. Given that the pre-set criterion of negligible correlations between models was met, models were considered separately. Based on the decision tree, individual parameters were generated by using final parameter estimates for the ALC model and resampled parameters for the RTTE model of RR, to account for uncertainty of the estimates.

Simulation of ALC dynamics was first performed. The percentages of virtual patients within each lymphopenia grade at the end of Y1 were comparable with the percentages observed in the CLARITY trial (refer to Electronic Supplementary Material 3, Table 1). The study size was then increased by blocks of 2000 patients, but this did not provide a better estimation as percentages were already stabilised on values obtained using the initial study size.

The occurrence of qualifying relapses for virtual patients was then simulated with the RTTE model of RR. The percentages of virtual patients not experiencing between 1 and 
Fig. 1 Distribution of sampled covariates. a Probability distribution of experiencing a different total number of relapses in the 12 months preceding study entry in simulated and observed patients. b Probability density functions of both resampled and observed creatinine clearance and body weight for female (top) and male (bottom) subjects. The density of each resampled covariate (green area) well reproduces the correspondent observed density (orange area), as shown by area overlapping and very similar mean values (dashed lines). $B W$ body weight, $C L_{C R}$ creatinine clearance, $E X N B$ number of relapses in the 12 months preceding study entry (a)

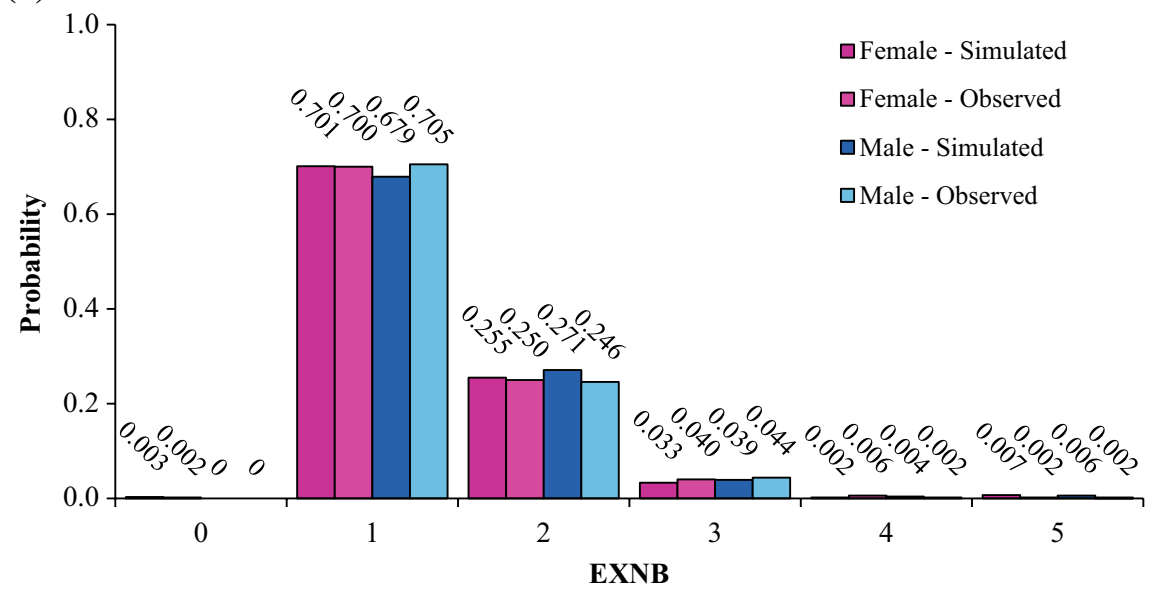

(b)

FEMALE
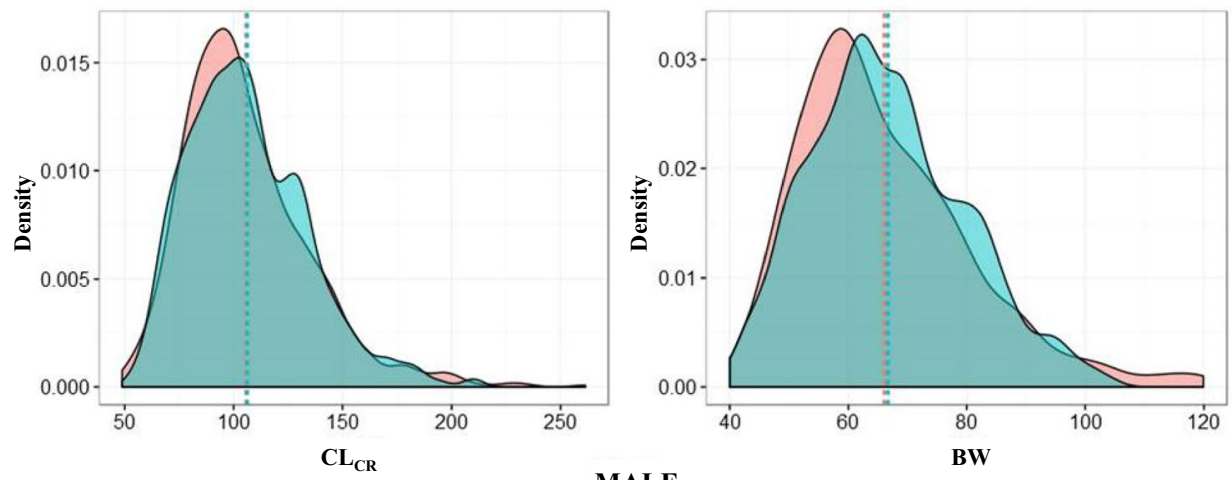

MALE

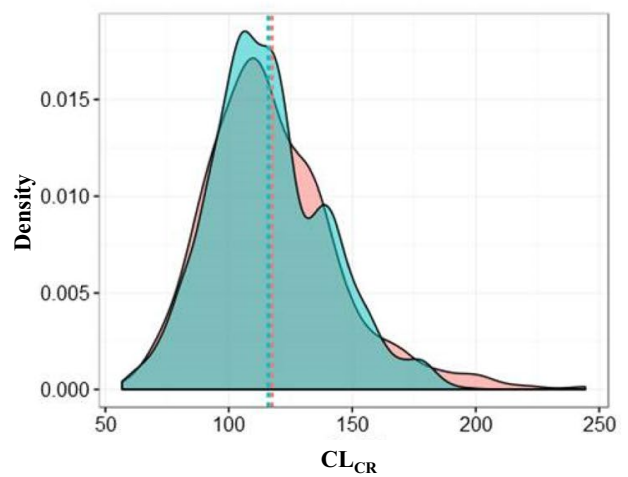

6 relapses by the end of $\mathrm{Y} 1$ and $\mathrm{Y} 2$ were comparable with those observed in patients treated with a Cladribine Tablets $3.5 \mathrm{mg} / \mathrm{kg}$ cumulative dose in the CLARITY study (refer to Electronic Supplementary Material 3, Table 2). The simulation framework could therefore be considered valid within its context of use.

\subsection{Proportion of Patients with Postponed Treatment in Year 2}

Following the assessment of the simulation workflow, the base scenario and treatment scenarios were simulated as described. Individual parameters obtained from model simulation under the CLARITY scenario along with resampled patient characteristics were preserved across scenarios to allow comparisons among the tested treatment rules.

Proportions of patients were similar across treatment scenarios that allowed postponements in patients with Grade 2-4 lymphopenia at the beginning of the second year, regardless of whether postponement blocks were 1 , 2 or 3 months (refer to Electronic Supplementary Material 3 , Table 3). In particular, the majority of virtual patients $(\sim 92 \%)$ required no postponement of treatment. Few patients $(\sim 7 \%)$ required 1 to 3 treatment postponements 
(postponement of 1-9 months in duration depending on the dosing algorithm) before starting the second year of treatment, and $<1 \%$ discontinued due to lymphopenia (i.e. they did not recover to Grade 0 or 1 after the last postponement, and hence did not receive the second-year treatment course). Results from scenario 2, consisting of 2-month blocks, are reported in Fig. 2.

Fewer instances of severe (Grade 3-4) lymphopenia were observed at any time during Y2 when a treatment algorithm was applied than in the base scenario, supporting treatment guidelines in preventing the potential risk associated with the development of severe lymphopenia. In those patients who qualified for postponements ( $8 \%$ of virtual patients), the proportion reaching Grade 3-4 lymphopenia at some time in the study was decreased (from 85 to $76 \%$ for treatment scenario 2) when the mitigation rule was applied (refer to Electronic Supplementary Material 3, Table 3).

\subsection{Effect of Treatment Rules on Relapse Rate}

There was a negligible effect of treatment rules on the probability of experiencing a qualifying relapse in the 2-year study, regardless of which postponement scenario was used. The probability distribution of predicted relapse-free survival is shown for both the base scenario and treatment scenario 2 in Fig. 3. In addition, the relapse-free survival Kaplan-Meier curves obtained for both scenarios are shown in Electronic Supplementary Material 3, Figures 2 and 3. Differences in relapse outcomes appear very small, suggesting that treatment postponements during Y2 do not lead to a clinically meaningful loss of efficacy. In Electronic Supplementary Material 3, Table 4, the proportions of subjects not experiencing 1 to 6 relapses at the end of $\mathrm{Y} 2$ are reported for the base and treatment scenarios. The model predictions obtained for the effect compartment exposure, hazard, cumulative risk for experiencing a first relapse over time, as well as the model-predicted relapse-free survival (i.e. 1 - cumulative risk) for a typical patient (weighing $69.3 \mathrm{~kg}$, with a $\mathrm{CL}_{\mathrm{CR}}$ of $104.5 \mathrm{~mL} / \mathrm{min}$, and an EXNB of 1) receiving Cladribine Tablets at a total cumulative dose of $3.5 \mathrm{mg} / \mathrm{kg}$ over 2 years according to different treatment postponement durations are shown in Fig. 4.
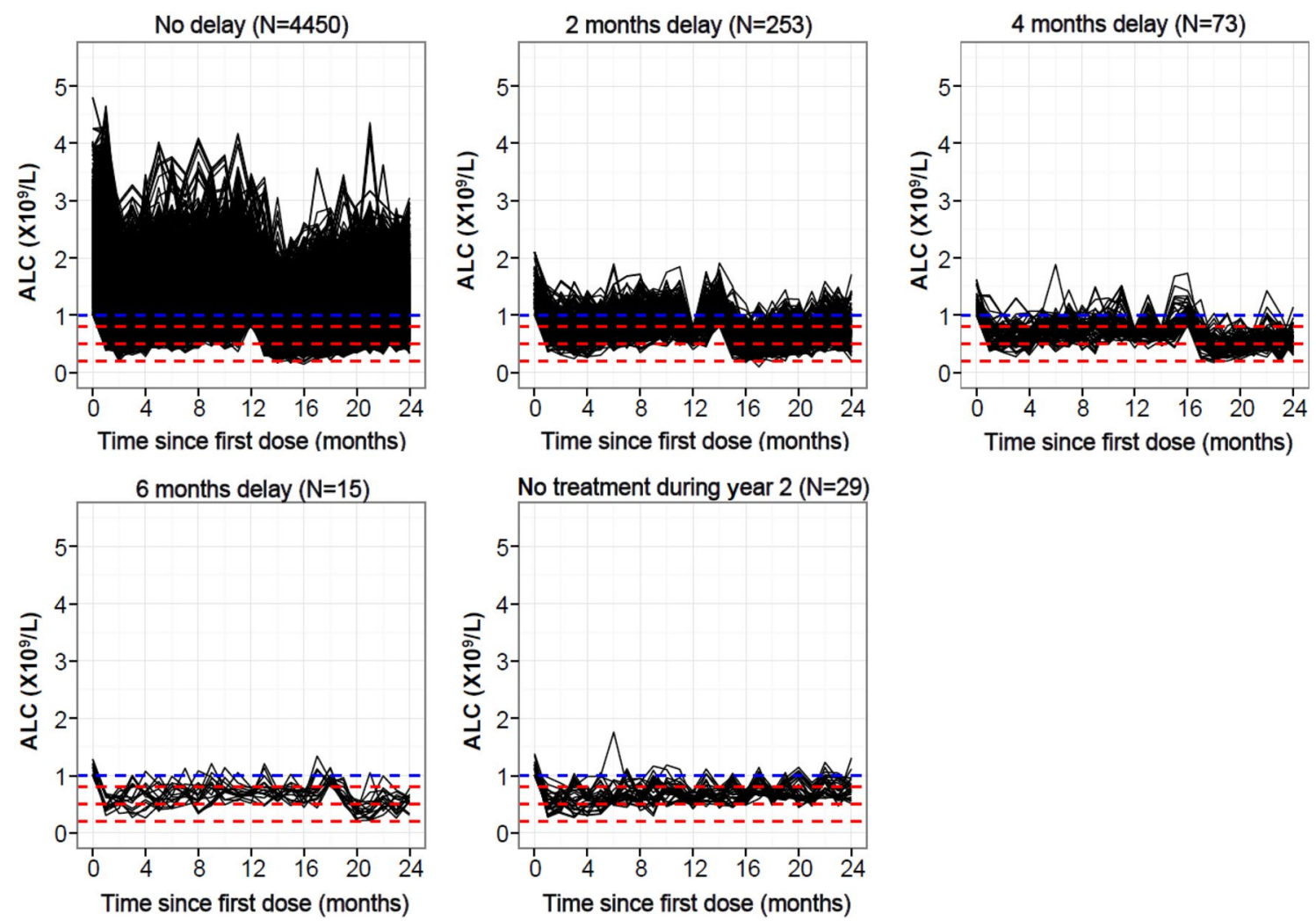

Fig. 2 Absolute lymphocyte count time-course profiles (treatment scenario 2). The blue dashed line denotes the upper limit for Grade 1 lymphopenia $\left(1.0 \times 10^{9}\right.$ cells $\left./ \mathrm{L}\right)$. The red dashed lines denote upper limits for Grades 2, 3 and 4 lymphopenia $(0.8,0.5$ and $0.2 \times 10^{9}$ cells $/ \mathrm{L}$, respectively). $A L C$ absolute lymphocyte count 


\section{\begin{tabular}{l|l|l|l|l|l|l|l|l|l|l} 
Probability & 0.9 & 0.8 & 0.7 & 0.6 & 0.5 & 0.4 & 0.3 & 0.2 & 0.1
\end{tabular}}
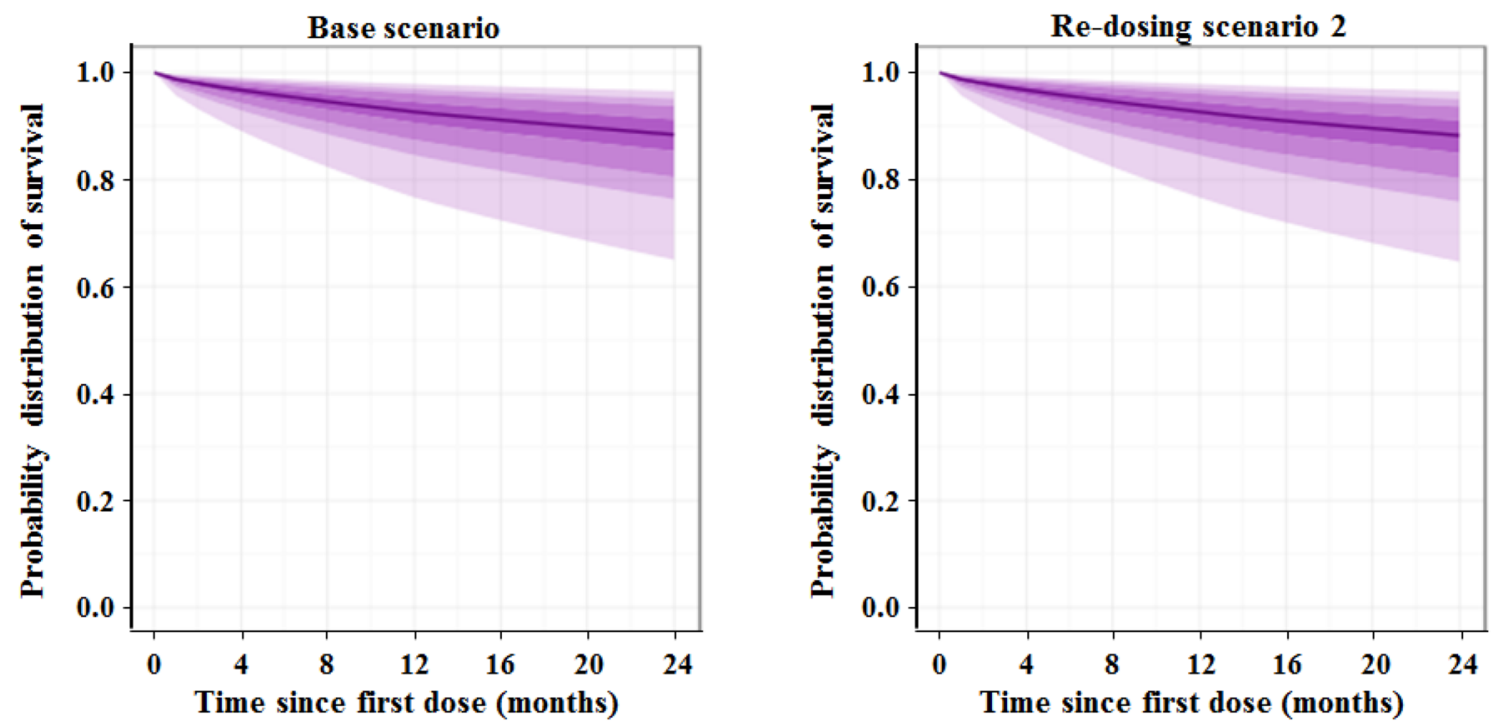

Fig. 3 Probability distribution of predicted relapse-free survival for percentiles from 10 to $90 \%$ shown over 24 months for the base scenario (left panel) and treatment scenario 2 (right panel)
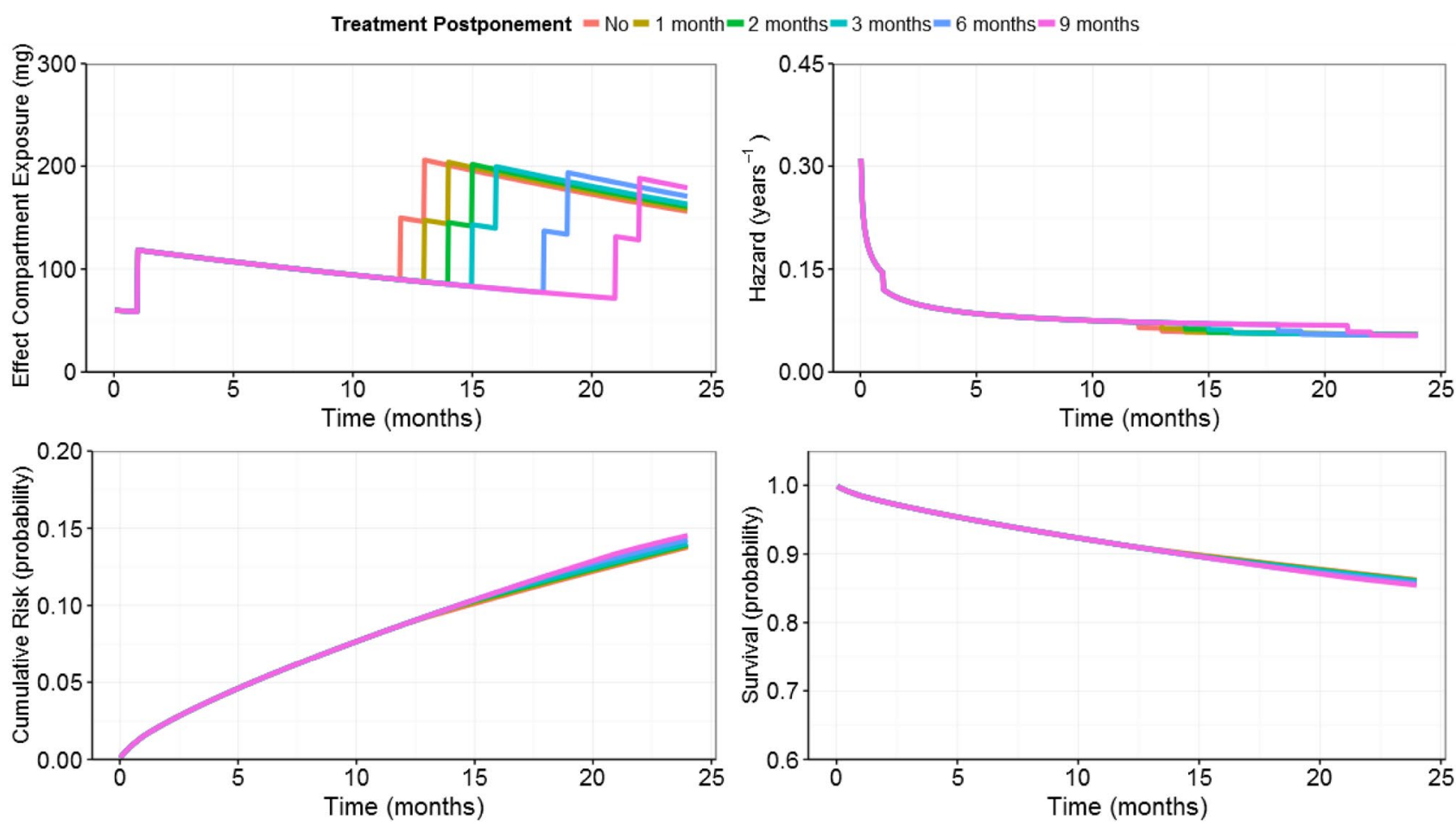

Fig. 4 Repeated time-to-event model predictions of relapse risk for the typical patient (weight $=69.3 \mathrm{~kg}$; creatinine clearance $=104.5 \mathrm{~mL} / \mathrm{min}$; number of relapses in the 12 months preced-

ing study entry $=1$ ) treated without postponement and with postponements of different month blocks (from 1 month up to 9 months) during year 2 


\section{Discussion}

The objective of this analysis was to investigate, in patients with RRMS qualifying for treatment postponement (i.e. patients with Grade 2-4 lymphopenia at the beginning of Y2), the impact of postponement or discontinuation of treatment with Cladribine Tablets in the second year on the time to occurrence of a qualifying relapse. Given that Grade 2-4 lymphopenia after cladribine treatment affects only a small proportion of patients, this is difficult to achieve as part of a clinical study. Instead, a clinical trial simulation approach was chosen so that multiple treatment scenarios could be tested based on data from the same virtual patient population [11].

Treatment in Y2 could be postponed 3 times in blocks of between 1 and 3 months in duration. The proportions of patients requiring any postponement or discontinuation of treatment during the second year were similar across considered treatment scenarios. In particular, in scenarios that permitted treatment postponements in patients with Grade 2-4 lymphopenia, approximately $92 \%$ of virtual patients would require no delay in the second-year course of Cladribine Tablets. An additional 7\% of patients would start the second year of cladribine treatment within 3 postponements, and less than $1 \%$ of patients would require treatment suspension because their ALC did not recover to $\geq 0.8 \times 10^{9}$ cells/L within 3 simulated postponements. These simulation results are in agreement with the results from an analysis of the CLARITY and CLARITY Extension studies that retrospectively applied the ALC-based treatment guidelines to patients that had received a Cladribine Tablets $7 \mathrm{mg} / \mathrm{kg}$ cumulative dose over 4 years (in CLARITY and CLARITY Extension, 1 year was defined as 48 weeks). At the end of the first year, $89 \%$ of patients had Grade $0-1$ lymphopenia and would therefore still have met the criteria for treatment in the second year (without any postponement) had the ALCbased treatment guidelines been enforced in these trials [12].

The appropriateness of the treatment guidelines in minimising the potential risk of developing severe lymphopenia was further confirmed by the observation of less Grade 3-4 lymphopenia at any time during Y2 when applying the redosing algorithms. Specifically, in the $8 \%$ of virtual patients qualifying for postponement (i.e. with Grade 2-4 lymphopenia at the beginning of $\mathrm{Y} 2$ ), the proportion of patients not experiencing Grade 3 or 4 lymphopenia during Y2 was increased when the mitigation rule was applied; this gain ranged from 8 to $12 \%$, depending on the postponement block definition (refer to Electronic Supplementary Material 3, Table 3). Following the RTTE model simulations for each considered scenario, practically no impact of treatment rules was observed on the mean predicted relapse-free survival and its probability distribution over time. Furthermore, comparable percentages of patients not experiencing a certain number of relapses (between 1 and 6) were obtained across the various scenarios.

In clinical practice, the decision to initiate treatment with Cladribine Tablets in Y1 and in Y2 is now based on ALC guidelines to minimise the risk of severe sustained lymphopenia [2]. It is recommended that ALC be measured prior to the start of treatment with Cladribine Tablets in each treatment year, and 2 and 6 months after start of treatment in each treatment year. If ALC is observed below $0.5 \times 10^{9}$ cells/L, ALC should be actively monitored until values increase. Treatment guidelines within the European Summary of Product Characteristics for Cladribine Tablets recommend delaying the second-year course of treatment for up to 6 months in patients whose ALC has not recovered to at least $0.8 \times 10^{9}$ cells/L (Grade 1 lymphopenia or better) [2]. A treatment course consists of 2 weekly treatments separated by 1 month. Thus, regardless of whether the first weekly treatment of Y2 has to be postponed due to lymphopenia or not, the subsequent treatment week always follows the first treatment week by 1 month.

\section{Conclusion}

ALC-based treatment guidelines are supported by the results of the analysis presented here. Our results indicate that $92 \%$ of patients will require no delay of the second-year course of treatment, but by allowing a 6-month delay, 99\% of patients would be eligible for their second-year course of treatment. Implementation of ALC-based treatment guidelines would reduce the occurrence of Grade 3-4 lymphopenia at any time, without having a negative impact on the risk of relapse over 2 years.

Acknowledgements The authors would like to thank the patients, investigators and co-investigators involved in the Cladribine Tablets clinical trial programme, as well as study teams at the participating centres, at Merck KGaA, Darmstadt, Germany, and at Merck Serono S.A., Switzerland. The authors thank the following people for their contribution to the analyses reported in this manuscript: Pascal Girard at Merck Serono S.A., Switzerland; Professor Marc Lavielle at Inria, France; and the Pharmacometrics group at Uppsala University, Sweden. The authors acknowledge Duncan Marriott of inScience Communications, Chester, UK, for medical writing support, funded by Merck KGaA, Darmstadt, Germany.

Funding The studies summarised in this report were sponsored by EMD Serono Inc., a business of Merck KGaA, Darmstadt, Germany (in the USA), and Merck Serono SA, Geneva, an affiliate of Merck KGaA, Darmstadt, Germany (rest of world). Medical writing assistance was funded by Merck KGaA, Darmstadt, Germany. 


\section{Compliance with Ethical Standards}

Conflict of interest Nadia Terranova and Alain Munafo are employees of Merck Serono SA, Switzerland, an affiliate of Merck KGaA, Darmstadt, Germany. Christine Hicking is an employee of Merck KGaA, Darmstadt, Germany. Fernando Dangond is an employee of EMD Serono, Inc., a business of Merck KGaA, Billerica, MA, USA.

Open Access This article is distributed under the terms of the Creative Commons Attribution-NonCommercial 4.0 International License (http://creativecommons.org/licenses/by-nc/4.0/), which permits any noncommercial use, distribution, and reproduction in any medium, provided you give appropriate credit to the original author(s) and the source, provide a link to the Creative Commons license, and indicate if changes were made.

\section{References}

1. Compston A, Coles A. Multiple sclerosis. Lancet. 2008;372(9648):1502-17.

2. Merck Serono Europe Limited. MAVENCLAD $10 \mathrm{mg}$ tablets summary of product characteristics. 2017. http://www.ema.europ a.eu/docs/en_GB/document_library/EPAR_-_Product_Informatio n/human/004230/WC500234561.pdf. Accessed 28 June 2018.

3. Cook S, Leist T, Comi G, Montalban X, Sylvester E, Hicking C, et al. Infections during periods of grade 3 or 4 lymphopenia in patients taking cladribine tablets $3.5 \mathrm{mg} / \mathrm{kg}$ : data from an integrated safety analysis. Mult Scler. 2017;23(S3):599.

4. National Cancer Institute. Common Terminology Criteria for Adverse Events v3.0 (CTCAE). 2006. https://ctep.cancer.gov/ protocoldevelopment/electronic_applications/docs/ctcaev3.pdf. Accessed 28 June 2018.
5. Giovannoni G, Comi G, Cook S, Rammohan K, Rieckmann $\mathrm{P}$, Soelberg Sorensen P, et al. A placebo-controlled trial of oral cladribine for relapsing multiple sclerosis. N Engl J Med. 2010;362(5):416-26.

6. Giovannoni G, Soelberg Sorensen P, Cook S, Rammohan K, Rieckmann P, Comi G, et al. Safety and efficacy of cladribine tablets in patients with relapsing-remitting multiple sclerosis: results from the randomized extension trial of the CLARITY study. Mult Scler. 2017:1352458517727603.

7. Novakovic AM, Thorsted A, Schindler E, Jönsson S, Munafo A, Karlsson MO. Pharmacometric analysis of the relationship between absolute lymphocyte count and expanded disability status scale and relapse rate efficacy endpoints in multiple sclerosis trials. J Clin Pharmacol. Epub. 2018. https://doi.org/10.1002/ jcph.1136.

8. Savic RM, Novakovic AM, Ekblom M, Munafo A, Karlsson MO. Population pharmacokinetics of cladribine in patients with multiple sclerosis. Clin Pharmacokinet. 2017;56(10):1245-53.

9. R Foundation for Statistical Computing. mlxR 2.2.0. 2015. https ://cran.r-project.org/src/contrib/Archive/mlxR/. Accessed 28 June 2018.

10. Lixoft. Mlxtran. Antony: Lixoft; 2015.

11. Holford N, Ma SC, Ploeger BA. Clinical trial simulation: a review. Clin Pharmacol Ther. 2010;88(2):166-82.

12. Cook S, Comi G, Giovannoni G, Rieckmann P, Soelberg-Sorensen $\mathrm{P}$, Vermersch $\mathrm{P}$, et al. Year-by-year lymphopenia rates in patients with relapsing multiple sclerosis (RMS) treated with cladribine tablets $3.5 \mathrm{mg} / \mathrm{kg}$ in CLARITY and re-treated in CLARITY Extension. Eur J Neurol. 2017;24(Suppl. 1):195.

13. National Cancer Institute. Common Terminology Criteria for Adverse Events v3.0 (CTCAE). Bethesda: National Cancer Institute; 2006. 